\title{
Statistical mechanics of the coagulation-diffusion process with a stochastic reset
}

\author{
Xavier Durang ${ }^{a}$, Malte Henkel $^{b}$ and Hyunggyu Park H $^{a}$ \\ ${ }^{a}$ School of Physics, Korea Institute for Advanced Study, Seoul 130-722, Korea \\ ${ }^{b}$ Groupe de Physique Statistique, Département de Physique de la Matière et des Matériaux, \\ Institut Jean Lamour (CNRS UMR 7198), Université de Lorraine Nancy, B.P. 70239, \\ F - 54506 Vandouvre lès Nancy Cedex, France
}

\begin{abstract}
The effects of a stochastic reset, to its initial configuration, is studied in the exactly solvable one-dimensional coagulation-diffusion process. A finite resetting rate leads to a modified non-equilibrium stationary state. If in addition the input of particles at a fixed given rate is admitted, a competition between the resetting and the input rates leads to a non-trivial behaviour of the particle-density in the stationary state. From the exact inter-particle probability distribution, a simple physical picture emerges: the reset mainly changes the behaviour at larger distance scales, while at smaller length scales, the non-trivial correlation of the model without a reset dominates.
\end{abstract}

PACS numbers: 05.40-a, 02.50-r, 87.23.Cc 


\section{Introduction}

Stochastic resets occur quite commonly in very distinct situations. For example, consider a network of tidal channels on a beach. From time to time, it is washed out by a larger wave. What would be the average properties of such a network, and how do they differ close to the water line (when resetting due to waves is frequent), and farther inland ? Another often-met instance concerns when searching for some object. A frequently-used search strategy consists in, after having searched in vain for some time, to return to the beginning and to start afresh, until the object is found. In remarkable work, Evans and Majumdar (EM) [15] have explored the consequences of stochastic resetting in simple diffusion of a single random walker. They considered a random walk on the line, with a time-dependent position $x(t)$ and starting from some initial position $x(0)=x_{0} \neq 0$, and also with an absorbing trap at the origin $x=0$. While undergoing the random walk, the particle is reset to its initial position with a rate $r$. EM showed that the statistical properties of the random walk are drastically altered by the resetting. For example, in the long-time limit, the stationary distribution of the particle with reset is no longer gaussian and the mean time to find a target at the origin becomes finite whenever $r>0$ and actually has a minimum at some non-trivial value $r^{*} \neq 0$ [15]. Various aspects of searches with reset have been analysed recently [2, 8, 28, 26, 33, 4] and these considerations have also been extended to the consideration of teams of independent researchers [27, 16, 17, 18].

Here, we are interested in analysing a simple model of interacting particles, subject to a stochastic reset to its initial configuration. We shall choose here the coagulation-diffusion process, which in one spatial dimension is exactly solvable through the method of empty intervals [5] and whose properties have been analysed profoundly in the past, e.g. [3, 6, 17, 9, 10, 11, 12, 13, 14, 21, 23, 25, 29, 30, 32, 35, 36]. The coagulation-diffusion process can be defined in terms of particles which move diffusively on an infinite chain such that each site is either empty or occupied by a single particle. If a particle makes an attempt to jump to a site which is already occupied, it disappears form the system with probability one, according to $A+A \rightarrow A$. As it is well-known, this system can be exactly solved through the method of empty intervals, where the central quantity is the probability $E_{n}(t)$ that $n$ consecutive sites are empty at time $t$. The time-dependent average particle-density is then given by $\rho(t)=\left(1-E_{1}(t)\right) / a$, whereas the $E_{n}$ satisfy for all $n \geq 1$ the equation [6, 9, 11]

$$
\partial_{t} E_{n}(t)=\frac{2 D}{a^{2}}\left(E_{n-1}(t)+E_{n+1}(t)-2 E_{n}(t)\right) \quad, \quad E_{0}(t)=1 \quad, \quad E_{\infty}(t)=0
$$

where $a$ is the lattice constant and $D$ the diffusion constant. In the continuum limit, one has $E_{n}(t) \rightarrow E(t, x)$, the particle-density becomes $\rho(t)=-\left.\partial_{x} E(t, x)\right|_{x=0}$ and finally (1.1) turns into $\left(\partial_{t}-2 D \partial_{x}^{2}\right) E(t, x)=0$ with the boundary conditions $E(t, 0)=1, E(t, \infty)=0$. It has been understood not so long ago how to treat these boundary conditions directly [13]. The resulting long-time behaviour $\rho(t) \sim t^{-1 / 2}$ has been confirmed in several experiments involving excitons moving on polymer chains [24, 22] or carbon nanotubes [31, 34]. The strong mathematical similarity of these equations with the ones for the probability distribution of a random walker [15] initially motivated us to consider a stochastic reset in the coagulation-diffusion process.

We now define the coagulation-diffusion process with a stochastic $\mathbf{r}$ eset (CDPR): consider a chain with $\mathcal{N}$ sites, each of which is can be occupied by at most one particle. The particles perform random hoppings to nearest-neighbour sites such that upon encounter of two particles, the arriving particle disappears. The stochastic reset is described by a given set of probabilities 


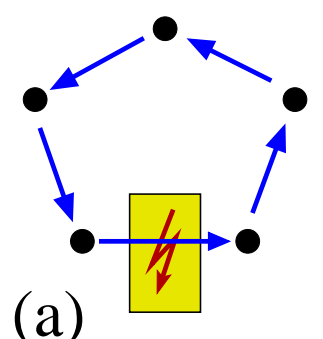

(a)



(b)

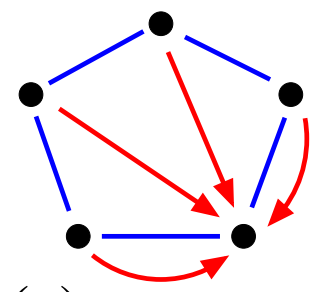

(c)

Figure 1: Schematic illustration of different kinds of non-equilibrium stationary states. The probability currents are indicated by the arrows. (a) Closed loop of probability currents, driven through the coupling to external engines. (b) Absorbing stationary state (red dot). (c) Network of probability currents, modified through a reset to a certain configuration, with the additional probability currents being indicated by red arrows.

$F_{n}$ for having $n$ consecutive empty sites 1 A sweep of the lattice consists of $\mathcal{N}$ steps of the microscopic dynamics. In each step, one chooses first a particle. This particle either diffuses with probability $\mathcal{P}_{g}=\frac{D}{2 D+r / \mathcal{N}}$ to the left, or else with probability $\mathcal{P}_{d}=\mathcal{P}_{g}$ to the right or finally the entire system is reset to the configuration $F_{n}$ with probability $\mathcal{P}_{r}=\frac{r / \mathcal{N}}{2 D+r / \mathcal{N}}$. In terms of the empty-interval probabilities $E_{n}(t)$, the equation of motion (1.1) is modified as follows by the stochastic reset

$$
\partial_{t} E_{n}(t)=\frac{2 D}{a^{2}}\left(E_{n-1}(t)+E_{n+1}(t)-2 E_{n}(t)\right)-r E_{n}(t)+r F_{n}, \quad E_{0}(t)=1 \quad, \quad E_{\infty}(t)=0
$$

which generalises the problem of the stochastic resetting of a single random walker as formulated by EM [15].

Besides being a case study of the influences of a stochastic reset, the results of this study might be also considered from a different point of view, by studying how a non-equilibrium system may be set up. A very common way is to allow for non-vanishing probability currents between the states of the system (which can be physically realised through coupling to external reservoirs), leading to closed loops between states, see figure 13. For a detailed review of the properties of the non-equilibrium stationary states, see [37] and references therein. An alternative possibility occurs for absorbing stationary states, where the system's evolution goes towards a single absorbing state it cannot leave anymore (figure 1b), see e.g. [20] for an overview and references therein. Here, and following EM, we consider what might be happening if the transition probabilities between states are changed such that the system can return with a certain probability $r$ to its initial state, see figure 1r.

This paper is organised as follows. In section 2, the exact solution of eq. (1.2) is derived. On a discrete chain, a detailed comparison with direct numerical simulation of the CDPR establishes that (1.2) is indeed the correct analytical description of the coagulation-diffusion process with reset. As seen before by $\mathrm{EM}$ for the random walk with reset, not only the stationary density of single particles and pairs is non-vanishing whenever $r>0$, but also the entire probability distribution is modified, and this can be illustrated through the explicit expressions for the $E_{n}(t)$. As a preparation for later generalisation, the continuum limit of the same model is derived and leads to the same qualitative conclusions. In section 3 we extend the model by

\footnotetext{
${ }^{1}$ For example, for a configuration of uncorrelated particles such that each single site is occupied with probability $p$, one has $F_{n}=(1-p)^{n}$.
} 
admitting in addition the possibility of particle-input on the lattice, at a fixed rate $\lambda$. It turns out that input and reset interact in a rather non-intuitive way which leads to a complex and non-monotonous dependence of the stationary particle density on these parameters. This surprising result will be further illuminated in section 4. Indeed, the shape of the distribution of the size of the empty intervals between particles reproduces the non-trivial correlations of coagulation-diffusion (with or without input) for small intervals where as the distribution of larger empty intervals is governed by the choice of the reset. Conclusions are given in section 5 . An appendix discusses details of the choice of the transition rates in Monte Carlo simulations.

\section{Model}

The $1 D$ coagulation-diffusion process with a reset (CDPR), as defined in the introduction, can be treated analytically by introducing the empty-interval probability $E_{n}(t)$, see [5] and refs. therein. The equation of motion is given by (1.2). In this section, we shall first compute the $E_{n}(t)$ exactly on an infinite chain and derive from this the particle- and the pair-densities. A detailed comparison with a direct numerical simulation of the CDPR will illustrate that (1.2) gives indeed the correct analytical description. We shall also study the continuum limit of the model.

An useful alternative route towards the stationary solution of (1.2) starts from the timedependent eq. (1.1), without a reset. Then the Laplace transform $\bar{E}_{n}(r):=\int_{0}^{\infty} \mathrm{d} t e^{-r t} E_{n}(t)$, along with the initial condition $E_{n}(0)=r F_{n}$ and with the formal replacement $t \mapsto r$ obeys the stationary equation (1.2) with a vanishing left-hand side, and the boundary condition $\bar{E}_{0}(r)=r^{-1}$. Although we shall not follow this route here, this idea might become useful to study the effects of a reset in more general situations.

\section{$2.1 \quad$ Discrete case}

The solution to (1.2) together with the non-trivial boundary condition $E_{0}(t)=1$, can be derived by admitting an analytical continuation to negative indices, via $E_{-n}(t)=2-E_{n}(t)$ [13]. Similarly, we shall define $F_{-n}:=2-F_{n}$ for the resetting distribution. Then, the generating function

$$
G(z, t):=\sum_{m=-\infty}^{\infty} z^{m} E_{m}(t)
$$

obeys, because of (1.2), the equation

$$
\partial_{t} G(z, t)=\frac{2 D}{a^{2}}\left(z+\frac{1}{z}-\left(2+\frac{r a^{2}}{2 D}\right)\right) G(z, t)+r F(z) .
$$

where $F(z)=\sum_{m \in \mathbb{Z}} z^{m} F_{m}$ is the generating function of the resetting distribution $F_{n}$. Eq. (2.2) is almost automatically solved. Setting the lattice constant $a=1$ from now on, the full solution can be decomposed as $E_{n}(t)=E_{n}^{(1)}(t)+E_{n}^{(2)}(t)$, with the 'homogeneous' solution $\left(I_{n}\right.$ is a modified Bessel function [1])

$$
E_{n}^{(1)}(t)=\mathrm{e}^{-(4 D+r) t} \sum_{m=-\infty}^{\infty} E_{m}(0) I_{n-m}(4 D t)
$$


and the 'inhomogeneous' part

$$
E_{n}^{(2)}(t)=r \int_{0}^{t} \mathrm{~d} t^{\prime} \mathrm{e}^{-(4 D+r) t^{\prime}} \sum_{m=-\infty}^{\infty} F_{m} I_{n-m}\left(4 D t^{\prime}\right) .
$$

Using the analytical continuations $E_{-n}(0)=2-E_{n}(0)$ and $F_{-n}=2-F_{n}$, we obtain

$$
\begin{aligned}
E_{n}^{(1)}(t)= & \mathrm{e}^{-(4 D+r) t}\left[\sum_{m=1}^{\infty} E_{m}(0)\left(I_{n-m}(4 D t)-I_{n+m}(4 D t)\right)+2 \sum_{m=1}^{\infty} I_{n+m}(4 D t)+I_{n}(4 D t)\right] \\
E_{n}^{(2)}(t)= & r \int_{0}^{t} \mathrm{~d} t^{\prime} \mathrm{e}^{-(4 D+r) t^{\prime}}\left[\sum_{m=1}^{+\infty} F_{m}\left(I_{n-m}\left(4 D t^{\prime}\right)-I_{n+m}\left(4 D t^{\prime}\right)\right)\right. \\
& \left.+2 \sum_{m=1}^{\infty} I_{n+m}\left(4 D t^{\prime}\right)+I_{n}\left(4 D t^{\prime}\right)\right]
\end{aligned}
$$

It is straightforward to check that these $E_{n}(t)=E_{n}^{(1)}(t)+E_{n}^{(2)}(t)$ indeed solve the equations of motion and obey the required boundary condition. We also observe that only the $F_{n}$ with $n \geq 1$ enter into the final solution. Since $E_{n}^{(1)}(t)$ simply reproduces the well-known solution without a reset [35, 13], clearly $E_{n}^{(2)}(t)$ gives the contributions to the reset. This illustrates how the stochastic reset modifies the entire probability-distribution of the states in the CDPR.

With the knowledge of those empty-interval probabilities, one can derive the particle-density $\rho(t)=P(\bullet)$ and the pair-density $p(t)=P(\bullet \bullet)$ using the following relations

$$
\begin{aligned}
& \rho(t)=1-E_{1}(t) \\
& p(t)=1-2 E_{1}(t)+E_{2}(t) .
\end{aligned}
$$

The particle-density $\rho(t)$ reads

$$
\begin{aligned}
\rho(t)= & \mathrm{e}^{-(4 D+r) t}\left(I_{0}(4 D t)+I_{1}(4 D t)-\sum_{m=1}^{\infty} \frac{m E_{m}(0) I_{m}(4 D t)}{2 D t}\right) \\
& +r \int_{0}^{t} \mathrm{~d} t^{\prime} \mathrm{e}^{-(4 D+r) t^{\prime}}\left(I_{0}\left(4 D t^{\prime}\right)+I_{1}\left(4 D t^{\prime}\right)-\sum_{m=1}^{\infty} \frac{m F_{m} I_{m}\left(4 D t^{\prime}\right)}{2 D t^{\prime}}\right)
\end{aligned}
$$

and the pair-density $p(t)$ is given by

$$
\begin{aligned}
p(t)= & \mathrm{e}^{-(4 D+r) t}\left[I_{0}(4 D t)-I_{2}(4 D t)-2 \sum_{m=1}^{\infty} \frac{m E_{m}(0) I_{m}(4 D t)}{2 D t}\right. \\
& \left.+\sum_{m=1}^{\infty} E_{m}(0)\left(\frac{(m-1) I_{m-1}(4 D t)}{2 D t}+\frac{(m+1) I_{m+1}(4 D t)}{2 D t}\right)\right] \\
& +r \int_{0}^{t} \mathrm{~d} t^{\prime} \mathrm{e}^{-(4 D+r) t^{\prime}}\left[I_{0}\left(4 D t^{\prime}\right)-I_{2}\left(4 D t^{\prime}\right)-2 \sum_{m=1}^{\infty} \frac{m F_{m} I_{m}\left(4 D t^{\prime}\right)}{2 D t^{\prime}}\right. \\
& \left.+\sum_{m=1}^{\infty} F_{m}\left(\frac{(m-1) I_{m-1}\left(4 D t^{\prime}\right)}{2 D t^{\prime}}+\frac{(m+1) I_{m+1}\left(4 D t^{\prime}\right)}{2 D t^{\prime}}\right)\right]
\end{aligned}
$$



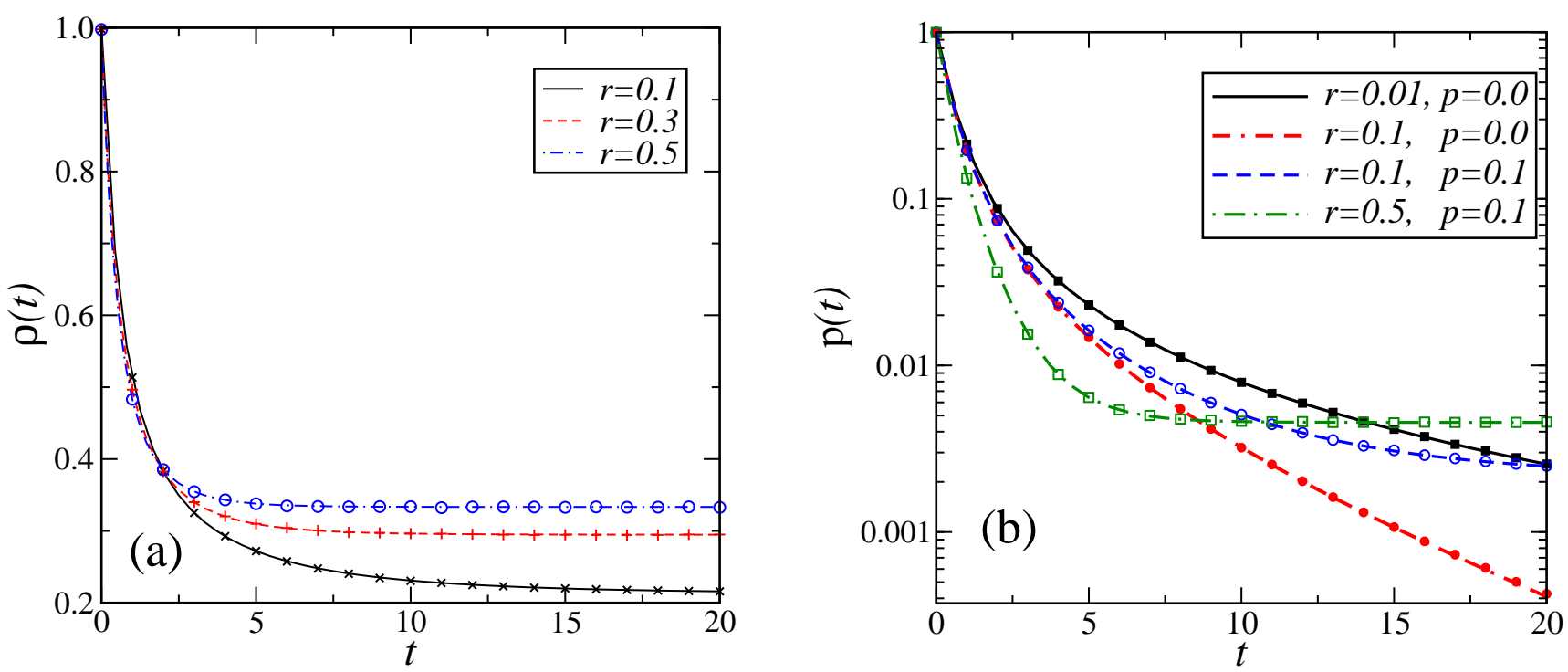

Figure 2: Left panel (a): particle-density $\rho(t)$ against time $t$ for different values of the reset parameter $r$ and particle concentration $p=0.5$. Right panel (b): pair-density $p(t)$ over against time for various values of the reset parameter $r$ and the particle concentration $p$. Here, an uncorrelated reset configuration $F_{n}=(1-p)^{n}$ was used. In both panels, the full line represents the analytic solution while the symbols show the Monte Carlo simulations.

Using several relations from [1], the stationary concentration is found from the second term of eq. (2.6)

$$
\begin{aligned}
\rho_{\text {stat }, \text { disc }}= & \frac{\sqrt{r(r+8 D)}-r}{4 D} \\
& -\frac{r}{2 D} \sum_{m=1}^{\infty}\left(\frac{1-p}{2}\right)^{m}\left(\frac{4 D}{r+4 D}\right)^{m}{ }_{2} F_{1}\left(\frac{m}{2}, \frac{m+1}{2} ; m+1 ;\left(\frac{4 D}{r+4 D}\right)^{2}\right)
\end{aligned}
$$

In figure 2, the analytic results (2.6|2.7) are compared with simulational results obtained from the CDPR as defined in section 1. Here, a reset to a random uncorrelated configuration of particles of mean concentration $p$, with an empty-interval distribution $F_{n}=(1-p)^{n}$, was used. We find a clear agreement which confirms the correctness of the equation of motion (1.2) and permits identification with the lattice model.

In the left panel of figure 2, the relaxation of the particle-density $\rho(t)$ towards its nonvanishing stationary value (since $p \neq 0$ and $r \neq 0$ ) is shown. One also sees that the relaxation towards the stationary value is exponentially fast, instead of the slow algebraic decay $\rho(t) \sim$ $t^{-1 / 2}$ obtained without reset. In the right panel of figure 2, an analogous behaviour is found for the pair-density $p(t)$ when the reset is made to a non-vanishing concentration, $p=0.1$. On the other hand, if one considers a reset to an empty lattice $(p=0)$, one clearly sees that the relaxation has become exponential, instead of the slow decay $p(t) \sim t^{-1}$ which would hold true in the absence of a reset. 


\subsection{Continuum limit}

In the continuum limit, the equation of motion (1.2) becomes

$$
\partial_{t} E(t, x)=2 D \partial_{x}^{2} E(t, x)-r E(t, x)+r F(x)
$$

with the boundary conditions $E(t, 0)=1$ and $E(t, \infty)=0$. One way to solve this equation is to separate the empty-interval probability as $E(t, x)=\frac{1}{2} f(x)+b(t, x)$ such that $f(x)$ will give the stationary solution and $b(t, x)$ will describe the relaxation towards it. Then, the equation for the stationary probability is

$$
f^{\prime \prime}(x)-\alpha^{2} f(x)+2 \alpha^{2} F(x)=0 ; f(0)=2, f(\infty)=0
$$

where

$$
\alpha^{2}:=\frac{r}{2 D} .
$$

The general solution of (2.10) is readily found by a variation of constants. Taking the two boundary conditions into account, a straightforward calculation gives

$$
f(x)=2 \mathrm{e}^{-\alpha x}+\alpha \int_{x}^{\infty} \mathrm{d} x^{\prime} F\left(x^{\prime}\right) \mathrm{e}^{\alpha\left(x-x^{\prime}\right)}+\alpha \int_{0}^{x} \mathrm{~d} x^{\prime} F\left(x^{\prime}\right) \mathrm{e}^{\alpha\left(x^{\prime}-x\right)}-\alpha \int_{0}^{\infty} \mathrm{d} x^{\prime} F\left(x^{\prime}\right) \mathrm{e}^{-\alpha\left(x+x^{\prime}\right)}
$$

To go further, a resetting distribution $F(x)$ must be specified. In the case of a random distribution where particles has a probability $p$ to be on a site, the empty-interval probability is given by $E_{n}(t)=(1-p)^{n}$ and, in the continuum limit $x=n a$ and $p \rightarrow 0$, the resetting distribution reads $F(x)=\mathrm{e}^{-c x}$, with the reset density $c=-\ln (1-p) \simeq p+\mathrm{O}\left(p^{2}\right)$. Then, the stationary part $f(x)$ is

$$
f(x)=\left(2-\frac{\alpha}{\alpha+c}\right) \mathrm{e}^{-\alpha x}+\frac{\alpha}{\alpha+c} \mathrm{e}^{-c x}+\frac{\alpha}{\alpha-c}\left(\mathrm{e}^{-(c-\alpha) x}-1\right) \mathrm{e}^{-\alpha x}
$$

such that the stationary concentration $\rho_{\text {stat }}$ is given by

$$
\rho_{\text {stat }}=-\left.\frac{1}{2} \frac{\partial f(x)}{\partial x}\right|_{x=0}=\frac{\alpha c}{\alpha+c} .
$$

It can be checked that in the limit $c \ll 1$ of small concentration, this expression is consistent with the discrete solution (2.8).

The dynamical part $b(t, x)$ of (2.9) satisfies the following equation

$$
\partial_{t} b(t, x)=2 D \partial_{x}^{2} b(t, x)-r b(t, x)
$$

with the boundary conditions $b(t, 0)=b(t, \infty)=0$. Using a sine Fourier transform, one can easily find the solution

$$
b(t, x)=\sqrt{\frac{\pi}{2 D t}} \mathrm{e}^{-2 D \alpha^{2} t} \int_{0}^{\infty} \mathrm{d} x^{\prime} b_{0}\left(x^{\prime}\right)\left[\mathrm{e}^{-\frac{\left(x-x^{\prime}\right)^{2}}{8 D t}}-\mathrm{e}^{-\frac{\left(x+x^{\prime}\right)^{2}}{8 D t}}\right]
$$

where $b_{0}(x)$ is the initial condition which can also be decomposed as $b_{0}(x)=E_{0}(x)-\frac{1}{2} f(x)$ where $f(x)$ will give the universal term and $E_{0}(x)$ will give the initial-condition-dependent term. Replacing $f(x)$ by its expression in (2.13), the universal term of the concentration reads

$$
\begin{aligned}
\rho_{\text {uni }}(t)= & -\left.\frac{\partial b_{\text {uni }}(x)}{\partial x}\right|_{x=0}=\sqrt{\frac{2 \pi}{D t}} \mathrm{e}^{-2 D \alpha^{2} t} \\
& +\frac{2 \pi \alpha c}{c^{2}-\alpha^{2}} \mathrm{e}^{-2 D \alpha^{2} t}\left(\alpha \mathrm{e}^{2 c^{2} D t} \operatorname{erfc}(c \sqrt{2 D t})-c \mathrm{e}^{2 D \alpha^{2} t} \operatorname{erfc}(\alpha \sqrt{2 D t})\right) .
\end{aligned}
$$


The initial-condition-dependent term becomes in the special case of initially uncorrelated particles, when $E_{0}(x)=\mathrm{e}^{-c x}$,

$$
\rho_{\text {dep }}(t)=2 \pi c \mathrm{e}^{-2 D\left(\alpha^{2}-c^{2}\right) t} \operatorname{erfc}(c \sqrt{2 D t})-\sqrt{\frac{2 \pi}{D t}} \mathrm{e}^{-2 D \alpha^{2} t}
$$

Hence the full particle-density becomes

$$
\rho(t)=\rho_{\text {stat }}+\rho_{\text {uni }}(t)+\rho_{\text {dep }}(t) \stackrel{t \rightarrow \infty}{\simeq} \frac{\alpha c}{\alpha+c}+\mathrm{O}\left(t^{-1 / 2} \exp \left(-2 D \alpha^{2} t\right)\right)
$$

The introduction of the reset has led to a non-vanishing stationary particle-density $\rho_{\text {stat }}$. For a fixed value of the initial concentration $c, \rho_{\text {stat }}$ increases monotonously as a function of the reset rate $\alpha$. This is qualitatively analogous to the behaviour of a single random walk with reset [15]. Furthermore, the leading approach towards this new non-equilibrium stationary state is for $\alpha>0$ exponentially fast, but reverts in the limit $\alpha \rightarrow 0$ to the standard slow relaxation $\mathrm{O}(1 / \sqrt{t})$ for the coagulation-diffusion model without reset.

\section{Stochastic reset and input of particles}

We now extend the model and allow the deposition ('input') of particles on the lattice, with a fixed rate $\lambda$. Without the reset, this has been treated long ago [9, 5]. In view of the technical complexities, we shall only treat the case of the continuum limit, where the equation of motion of the empty intervals becomes, for $x \geq 0$

$$
\partial_{t} E(t, x)=2 D \partial_{x}^{2} E(t, x)-\lambda x E(t, x)-r E(t, x)+r F(x)
$$

and subject to the boundary and initial conditions

$$
E(t, 0)=1, E(t, \infty)=0, E(0, x)=E_{0}(x)
$$

Again, one may separate this as $E(t, x)=\frac{1}{2} f(x)+b(t, x)$ such that $f(x)$ will give the stationary solution and $b(t, x)$ will describe the relaxation towards it. Introducing the abbreviations

$$
\alpha^{2}:=\frac{r}{2 D}, \beta^{3}:=\frac{\lambda}{2 D}, \mu:=\frac{\alpha^{2}}{\beta^{3}}=\frac{r}{\lambda}
$$

the equation for the stationary empty-interval distribution becomes

$$
f^{\prime \prime}(x)-\beta^{3}(x+\mu) f(x)+2 \alpha^{2} F(x)=0 ; \quad f(0)=2, \quad f(\infty)=0
$$

This may be solved by the standard variation of constants, although the expressions become quite lengthy. The general solution of the homogeneous part of (3.4) is

$$
f_{\text {hom }}(x)=c_{1} \frac{\sqrt{3}}{2}(\operatorname{Bi}(\beta(x+\mu))-\sqrt{3} \operatorname{Ai}(\beta(x+\mu)))+c_{2} \pi \sqrt{3} \operatorname{Ai}(\beta(x+\mu))
$$


Table 1: Limit behaviour of the scaling function $u P(u, y)$ in eq. (3.8), for small and large values of $u$ and $y$, respectively.

\begin{tabular}{|l|ll|}
\hline$(2 \pi)^{-1} 3^{5 / 6} \Gamma(2 / 3)^{2}+3^{2 / 3} \Gamma(2 / 3)(4 \pi)^{-1}\left(3 \Gamma(2 / 3)^{3}-4 \pi^{2} / 3\right) y$ & $u \rightarrow 0$ & $y \rightarrow 0$ \\
$y^{-1}-u / c F^{\prime}(0)$ & $u \rightarrow 0$ & $y \rightarrow \infty$ \\
\hline$(2 \pi)^{-1} 3^{5 / 6} \Gamma(2 / 3)^{2}+(2 \pi)^{-2} 3^{5 / 3} \Gamma(2 / 3)^{3} y$ & $u \rightarrow \infty$ & $y \rightarrow 0$ \\
$y^{1 / 2}$ & $u \rightarrow \infty$ & $y \rightarrow \infty$ \\
\hline
\end{tabular}

where $\mathrm{Ai}$ and $\mathrm{Bi}$ are Airy functions [1] and $c_{1,2}$ are constants. Then the general solution of (3.4) can be written in the form

$$
\begin{aligned}
f(x)= & c_{1} \frac{\sqrt{3}}{2}(\operatorname{Bi}(\beta(x+\mu))-\sqrt{3} \operatorname{Ai}(\beta(x+\mu)))+c_{2} \pi \sqrt{3} \operatorname{Ai}(\beta(x+\mu)) \\
& +\frac{2 \alpha^{2}}{\beta}[\operatorname{Bi}(\beta(x+\mu))-\sqrt{3} \operatorname{Ai}(\beta(x+\mu))] \int_{x}^{\infty} \mathrm{d} x^{\prime} F\left(x^{\prime}\right) \operatorname{Ai}\left(\beta\left(x^{\prime}+\mu\right)\right) \\
& +\frac{2 \alpha^{2}}{\beta} \operatorname{Ai}(\beta(x+\mu)) \int_{0}^{x} \mathrm{~d} x^{\prime} F\left(x^{\prime}\right)\left[\operatorname{Bi}\left(\beta\left(x^{\prime}+\mu\right)\right)-\sqrt{3} \operatorname{Ai}\left(\beta\left(x^{\prime}+\mu\right)\right)\right]
\end{aligned}
$$

Using the asymptotic behaviour of the Airy functions [1], it is easy to see that $f(\infty)=0$ implies that $c_{1}=0$ and the second boundary condition $f(0)=2$ fixes $c_{2}$. This leads to

$$
\begin{aligned}
f(x)= & \frac{2 \operatorname{Ai}(\beta(x+\mu))}{\operatorname{Ai}(\beta \mu)}-\frac{2 \pi \alpha^{2}}{\beta} \frac{\operatorname{Bi}(\beta \mu)}{\operatorname{Ai}(\beta \mu)} \operatorname{Ai}(\beta(x+\mu)) \int_{0}^{\infty} \mathrm{d} x^{\prime} F\left(x^{\prime}\right) \operatorname{Ai}\left(\beta\left(x^{\prime}+\mu\right)\right) \\
& +\frac{2 \pi \alpha^{2}}{\beta} \operatorname{Bi}(\beta(x+\mu)) \int_{x}^{\infty} \mathrm{d} x^{\prime} F\left(x^{\prime}\right) \operatorname{Ai}\left(\beta\left(x^{\prime}+\mu\right)\right) \\
& +\frac{2 \pi \alpha^{2}}{\beta} \operatorname{Ai}(\beta(x+\mu)) \int_{0}^{x} \mathrm{~d} x^{\prime} F\left(x^{\prime}\right) \operatorname{Bi}\left(\beta\left(x^{\prime}+\mu\right)\right)
\end{aligned}
$$

\subsection{Stationary density of particles}

From the previous equation (3.6), the stationary density of particles is obtained, and which can be written down in a scaling form which also involves the average particle-density $c=-F^{\prime}(0)$ in the reset configuration $F(x)$, and reads

$$
\rho_{\text {stat }}=-\left.\frac{1}{2} \frac{\partial f(x)}{\partial x}\right|_{x=0}=c P\left(\frac{c}{\beta}, \beta \mu\right)
$$

with the explicit scaling function

$$
P(u, y):=-\frac{1}{u} \frac{\operatorname{Ai}^{\prime}(y)}{\operatorname{Ai}(y)}-\pi y\left(\operatorname{Bi}^{\prime}(y)-\operatorname{Ai}^{\prime}(y) \frac{\operatorname{Bi}(y)}{\operatorname{Ai}(y)}\right) \int_{0}^{\infty} \mathrm{d} Y F(u Y / c) \operatorname{Ai}(Y+y)
$$

Herein, the first scaling variable $u=c / \beta$ measures the ratio of the particle-density of the reset configuration with respect to the stationary density without reset and the second scaling variable $y=\beta \mu=(\alpha / \beta)^{2}$ is a function of the ratio of the reset rate with the input rate. The scaling function $P=\rho_{\text {stat }} / c$ itself measures directly the stationary density in units of the reset 



Figure 3: Plot of the scaling function $u P(u, y)$ as a function of $y$ and for different values of $u$. The left panel (a) uses the reset function $F(x)=\exp (-c x)$, appropriate for uncorrelated particles; the inset shows the same plot for the values $u=[0.75,0.80,0.85,0.90,0.95]$ from bottom to top. In the right panel (b), the scaling function $u P(u, y)$ for the choice $F(x)=\operatorname{erfc}\left(\frac{1}{2} \sqrt{\pi} c x\right)$ is shown for comparison.

density $c$. In table 1 the asymptotic behaviour of the scaling functions for $u$ and $y$ small or large are listed (remarkably, the limits are independent of the choice for the reset distribution $F(x))$. For $y \rightarrow 0$ one always recovers the known stationary particle density of the case without reset [9, 5], as expected. However, the qualitative behaviour of $P(u, y)$ as a function of $y$ changes according to the fixed value of $u$. When $u \ll 1, P(u, y)$ will monotonously decrease as a function of $y$, whereas for $u \gg 1$, one observes a monotonous increase. From table 1, this can be read off analytically from the small-y behaviour

$$
u P(u, y) \simeq \begin{cases}0.729-0.531 y & ; \text { if } u \rightarrow 0 \\ 0.729+0.392 y & ; \text { if } u \rightarrow \infty\end{cases}
$$

The surprisingly complex behaviour of $P(u, y)$ is further illustrated in figure 3 and also depends in a subtle way on the choice of the resetting configuration $F(x)$. We begin with the case $F(x)=e^{-c x}$ of uncorrelated particles with concentration $c$. From figure 3 a, it can be seen that there is also an intermediate range $u \approx 0.8-0.9$, when $P(u, y)$ is a non-monotonous function of $y$. From the inset in figure 3 a, it can be seen that $P(u, y)$ goes through a minimum before the final growing regime for $y$ sufficiently large is reached. A local analysis shows that $\partial P(u, y) / \partial y<0$ for $u=u_{c}:=\leq 0.9295765 \ldots$, which means that this minimum exists for all $u<u_{c}$.

For different choices of $F(x)$, one may encounter different scenarios. In figure $3 \mathrm{~b}$, we show results for the choice $F(x)=\operatorname{erfc}\left(\frac{1}{2} \sqrt{\pi} c x\right) 2$ Without neither a reset nor an input, the system will converge towards this distribution, with a certain (time-dependent) particle-density $c$.

\footnotetext{
${ }^{2}$ In the simple coagulation-diffusion process starting from an initially fully occupied lattice, this is the exact shape of the empty-interval probability $E_{n}(t)$ with a known time-dependent density $c=c(t)[13$.
} 

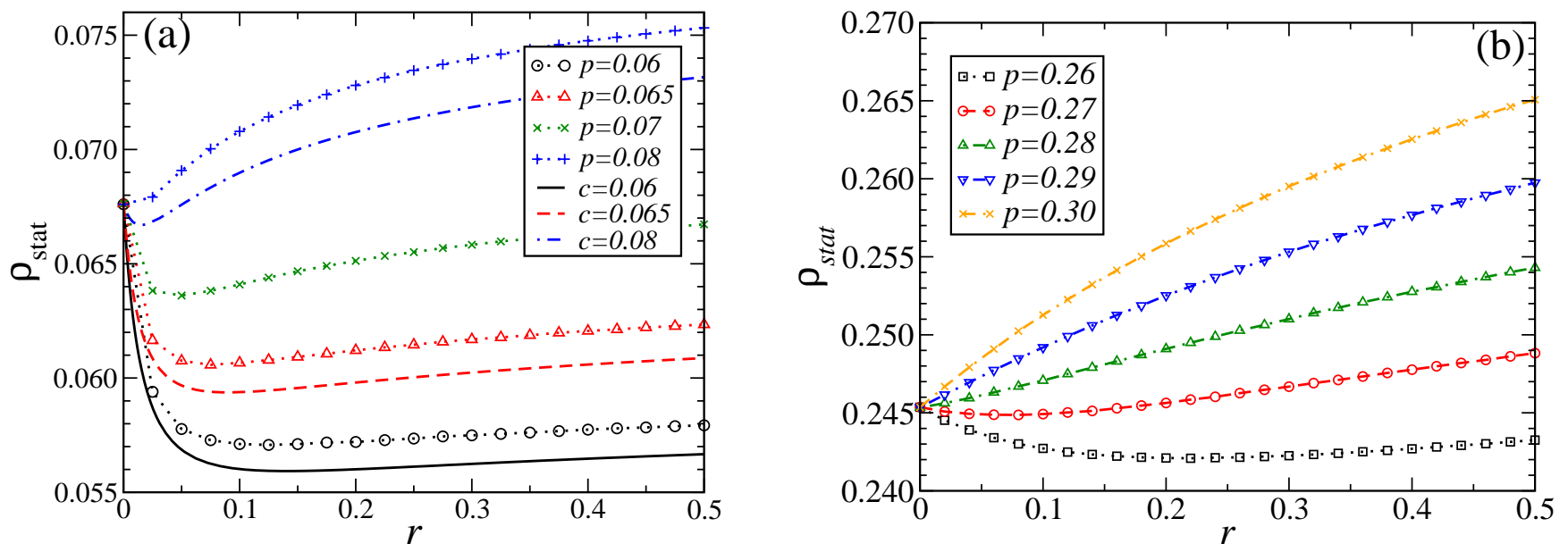

Figure 4: Plot of the stationary probability $\rho_{\text {stat }}$ as a function of the reset parameter $r$ towards uncorrelated particles for two values of the input parameter: (a) left panel $\lambda=0.0008$, (b) right panel $\lambda=0.04$. In the left panel, the full lines without symbols give the analytical solution (3.7) in the continuum limit.

While for $u \ll 1$ and for $u \gg 1$, the qualitative behaviour is analogous to the one seen before, a non-monotonous behaviour now occurs in the middle region $u \approx 0.7-0.75$, with $u_{c}=0.7010036 \ldots$ However, for $u$ not too far above $u_{c}$, the scaling function now rather shows a maximum.

The analytical results of this section were obtained in the continuum limit. In figure 4 , results from a direct numerical simulation of the CDPR with input are shown, for two values of the input rate $\lambda$, and in the case of a reset towards uncorrelated particles. Qualitatively, the behaviour of the stationary density is analogous to the one seen in figure 3 a and hence is in qualitative agreement with the analytic solution (3.7), obtained in the continuum limit. While both the discrete and the continuum versions of the CDPR lead to the same qualitative conclusions, the precise form of the stationary density is influenced by the fact that the simulations were carried out on a discrete chain.

\subsection{Dynamics}

Now, we complete this study by deriving the analytical solution for dynamical part $b(t, x)$ which satisfies

$$
\partial_{\tau} b(\tau, x)=\partial_{x}^{2} b(\tau, x)-\beta^{3} x b(\tau, x)-\alpha^{2} b(\tau, x)
$$

where time was rescaled according to $\tau:=2 D t$ and one also has the boundary and initial conditions

$$
b(\tau, 0)=b(\tau, \infty)=0, \quad b(0, x)=b_{0}(x)=2 E_{0}(x)-f(x)
$$

In principle, one may solve this by using a Laplace transform $\bar{b}(s, x)=\int_{0}^{\infty} e^{-s \tau} b(\tau, x)$. This gives the equation

$$
\partial_{x}^{2} \bar{b}(s, x)-\beta^{3} x \bar{b}(s, x)-\left(s+\alpha^{2}\right) \bar{b}(s, x)+b_{0}(x)=0
$$

along with the boundary conditions $\bar{b}(s, 0)=\bar{b}(s, \infty)=0$. This is the same type as eq. (3.4). An analogous straightforward, but just a little tedious, calculation leads to 
Table 2: The stationary empty-interval probability $E_{\text {stat }}(x)$ and the corresponding $\operatorname{IPDF} \mathcal{D}(x)$ for three types of systems: (a) uncorrelated particles, (b) coagulation-diffusion and (c) with additional particle input. The distributions are characterised by the model parameters $c$ and $\beta$.

\begin{tabular}{|ll|ll|}
\hline \multicolumn{1}{|c|}{ type } & $E_{\text {stat }}(x)$ & $\mathcal{D}(x)$ \\
\hline (a) & uncorrelated & $\exp (-c x)$ & $c \exp (-c x)$ \\
(b) & coagulation-diffusion & $\operatorname{erfc}\left(\frac{1}{2} \sqrt{\pi} c x\right)$ & $\frac{1}{2} \pi c^{2} x \exp \left(-\frac{\pi}{4} c^{2} x^{2}\right)$ \\
$(\mathrm{c})$ & with particle-input & $\mathrm{Ai}(\beta x) / \mathrm{Ai}(0)$ & $\beta^{2} x \mathrm{Ai}(\beta x) /\left|\mathrm{Ai}^{\prime}(0)\right|$ \\
\hline
\end{tabular}

$$
\begin{aligned}
& \bar{b}(s, x)=\frac{\pi}{\beta}\left\{-\int_{0}^{\infty} \mathrm{d} Y b_{0}(Y) \operatorname{Ai}\left(\beta(Y+\mu)+s / \beta^{2}\right) \operatorname{Ai}\left(\beta(x+\mu)+s / \beta^{2}\right) \frac{\operatorname{Bi}\left(\beta \mu+s / \beta^{2}\right)}{\operatorname{Ai}\left(\beta \mu+s / \beta^{2}\right)}\right. \\
& \left.+\int_{x}^{\infty} \mathrm{d} Y \operatorname{Ai}\left(\beta(Y+\mu)+s / \beta^{2}\right) \operatorname{Bi}\left(\beta(x+\mu)+s / \beta^{2}\right)+\int_{0}^{x} \mathrm{~d} Y \operatorname{Bi}\left(\beta(Y+\mu)+s / \beta^{2}\right) \operatorname{Ai}\left(\beta(x+\mu)+s / \beta^{2}\right)\right\}
\end{aligned}
$$

Generalising [32, the inverse Laplace transform is now formally found from the poles of $\bar{b}(s, x)$, which arise via the zeroes of the Airy function, in the first term. The result is

$$
b(t, x)=-\pi \beta \sum_{n=1}^{\infty} \int_{0}^{\infty} \mathrm{d} x^{\prime} b\left(0, x^{\prime}\right) \operatorname{Ai}\left(\beta x^{\prime}+a_{n}\right) \operatorname{Ai}\left(\beta x+a_{n}\right) \frac{\operatorname{Bi}\left(a_{n}\right)}{\operatorname{Ai}^{\prime}\left(a_{n}\right)} \exp \left(-t\left(r+\left|a_{n}\right| \beta^{2}\right)\right.
$$

where $a_{n}$ is the $n^{\text {th }}$ zero of the Airy function [1]. From this, one can read off the leading relaxation time $\tau_{\text {rel }}=1 /\left(\left|a_{1}\right| \beta^{2}+r\right)$, which is finite.

\section{Inter-particle distribution function}

In order to better appreciate the physical nature of the stationary state, we now study the properties of the inter-particle distribution function (IPDF), denoted here as $\mathcal{D}(x)$. On a discrete lattice $\mathcal{D}_{n}$ would be the probability that the nearest neighbour of a particle would be at a distance of $n$ sites. In the continuum limit, this becomes $\mathcal{D}(x)$. The relation to the stationary empty-interval probability $E_{\text {stat }}(x)=\frac{1}{2} f(x)$ is well-known [5]

$$
\mathcal{D}(x)=\frac{1}{2 \rho_{\text {stat }}} \frac{\partial^{2} f(x)}{\partial x^{2}}
$$

with the stationary density $\rho_{\text {stat }}$ found above. For the following discussion, we shall require the well-known expressions for $\mathcal{D}(x)$, as listed in table 2, for three paradigmatic systems; see e.g. [5] and references therein for the computational details.

Clearly, the case (a) of uncorrelated particles will be an important test case for the study of the effects of a reset. Recall that this distribution is also obtained for a reversible coagulationdiffusion process with the extra reaction $A \rightarrow A+A[5]$, such that the stationary state is an equilibrium state. The second case (b) describes the correlations spontaneously generated during a coagulation-diffusion process $A+A \rightarrow A$. In these two cases, the parameter $c$ denotes the average particle-density. It is known that for an arbitrary initial condition in pure 
coagulation-diffusion, the system converges towards this distribution, with an explicitly known time-dependent concentration $c=c(t)$ [35, 5, 13]. Finally, case (c) gives the stationary distribution with an additional input of particles. For later comparisons, recall the asymptotic form $\mathcal{D}(x) \stackrel{x \rightarrow \infty}{\sim} x^{3 / 4} \exp \left(-\frac{2}{3}(\beta x)^{3 / 2}\right)$.

It is clear from table 2 that the three systems are clearly distinguished via their IPDFs for large interval sizes $x \rightarrow \infty$. This observation will become the central tool in our analysis of the IPDF with a reset.

\subsection{IPDF without input}

Using the previous expression (2.12) of the function $f(x)$, and the definition (4.1), the IPDF can be cast into a scaling form

$$
\mathcal{D}(x)=\alpha D(\xi, v), \quad \xi:=c x \quad, \quad v:=\frac{\alpha}{c}
$$

with the explicit scaling function

$$
\begin{aligned}
& D(\xi, v)=\frac{\alpha}{\rho_{\text {stat }}}\left\{e^{-v \xi}-F\left(\frac{\xi}{c}\right)\right. \\
& \left.+\frac{v}{2}\left[\int_{0}^{\xi} \mathrm{d} Y F\left(\frac{Y}{c}\right) e^{v(Y-\xi)}+\int_{\xi}^{\infty} \mathrm{d} Y F\left(\frac{Y}{c}\right) e^{v(\xi-Y)}-\int_{0}^{\infty} \mathrm{d} Y F\left(\frac{Y}{c}\right) e^{-v(Y+\xi)}\right]\right\}
\end{aligned}
$$

In what follows, we shall discuss two specific examples: first, for a reset to uncorrelated particles with mean density $c$, one has $F(x)=e^{-c x}=e^{-\xi}$ and

$$
D_{(a)}(\xi, v)=\frac{\exp (-v \xi)-\exp (-\xi)}{1-v}
$$

Second, for a reset to a coagulation-diffusion configuration with density $c$, one has $F(x)=$ $\operatorname{erfc}\left(\frac{1}{2} \sqrt{\pi} \xi\right)$, hence

$$
D_{(b)}(\xi, v)=\frac{1}{2 \operatorname{erfc}(v / \sqrt{\pi})}\left[e^{-\xi v}\left(1+\operatorname{erf}\left(\xi \frac{\sqrt{\pi}}{2}-\frac{v}{\sqrt{\pi}}\right)\right)-e^{\xi v} \operatorname{erfc}\left(\xi \frac{\sqrt{\pi}}{2}+\frac{v}{\sqrt{\pi}}\right)\right]
$$

These functions are displayed in figure 5. First, in the left panel, the scaling function $D_{(a)}$ is shown. For the simple coagulation-diffusion process under study here, one would expect, consulting table 2(b), a gaussian shape of the IPDF. Clearly, this is no longer the case in the presence of a reset. Rather, one sees that although $D(\xi) \stackrel{\xi \rightarrow 0}{\rightarrow} 0$ as it should be for a stationary IPDF [5], for larger intervals one has an exponential distribution, typical of a system of uncorrelated particles 3 Furthermore, one observes that the effective density of particles in the large- $\xi$ regime (which can be read off from the slope of $\ln D(\xi)$ ) depends in a non-trivial way on the scaling parameter $v=\alpha / c$. Namely, if $v>1$, then the effective particle-density is simply unity, whereas if $v<1$, that effective particle-density is equal to $v$ (it remains to be seen whether this kind of dynamical transition also occurs in different models). In conclusion, the

\footnotetext{
${ }^{3}$ This is analogous to the finding of EM that the probability distribution of a random walk with reset is no longer gaussian.
} 

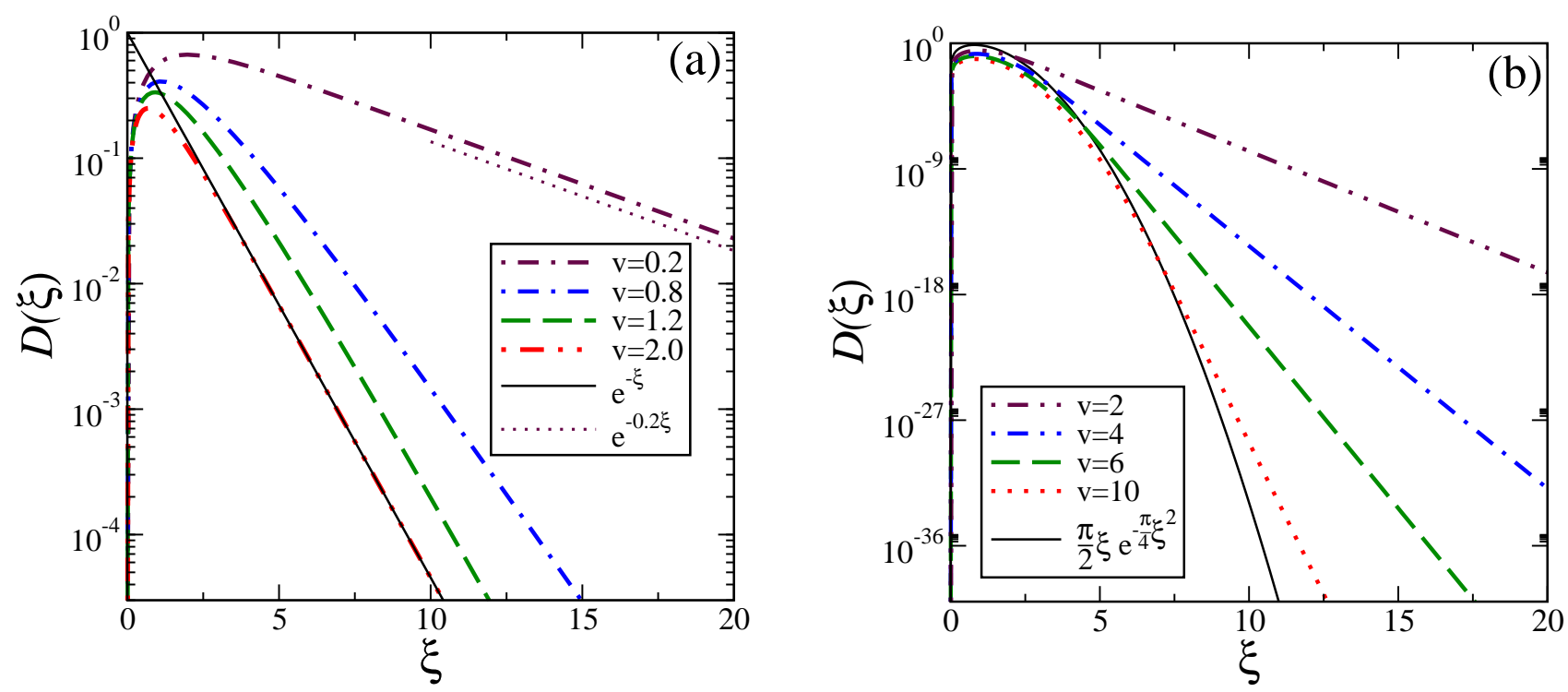

Figure 5: Stationary IPDF $D(\xi, v)$ of the coagulation-diffusion process, as a function of the scaled interval size $\xi$ and several values of the scaling variable $v$. Left panel (a): reset distribution $F(x)=\exp (-c x)$; the IPDF eq. (4.4) is shown for $v=[0.2,0.8,1.2,2.0]$ from top to bottom. The distributions $e^{-\xi}$ and $e^{-0.2 \xi}$ are also indicated. Right panel (b): reset distribution $F(x)=$ $\operatorname{erfc}\left(\frac{1}{2} \sqrt{\pi} c x\right)$; the IPDF eq. (4.5) is shown with $v=[2,4,6,10]$ from top to bottom. The distribution $\partial_{\xi}^{2} \operatorname{erfc}\left(\frac{\sqrt{\pi}}{2} \xi\right)=\frac{\pi}{2} \xi \mathrm{e}^{-\frac{\pi}{4} \xi^{2}}$ is also shown.

behaviour of the IPDF at small scales is quite distinct from the one seen at large scales. This is a consequence of the fact that the stationary state in the presence of a reset can no longer be described an equilibrium state.

A further aspect of this become apparent if a different reset configuration is analysed, see the right panel of figure 5 with the scaling function $D_{(b)}$. Here, the reset is done to a configuration of particles as obtained from an usual coagulation-diffusion process, with the natural correlations corresponding to a given density $c$. At first sight, one might expect that the reset to such a correlated configuration should lead to these correlations being maintained for all interval sizes $\xi$. However, it can be seen from figure 5 that this is not so. Rather, in the stationary state the correlated particle configurations only describe the actual stationary IPDF only at small interval sizes $\xi$. At larger sizes, one observes again an effective distribution corresponding to uncorrelated particles.

Intuitively, the observation from figure 5 b may be understood as follows: through the reset rate $r$, a further time scale $\tau_{r} \sim \alpha^{-2}$ is introduced which in turn creates a new length scale $\xi_{r} \sim \alpha$. Between two reset events, the system has on average enough time to reconstitute its natural correlations up to scales $\xi \lesssim \xi_{r}$ but since the resets are uncorrelated, beyond that scale its particles have become uncorrelated. The non-equilibrium nature of the stationary state manifests itself in strong short-distance correlations, as prescribed by the original dynamics, and an uncorrelated behaviour at large distances. 

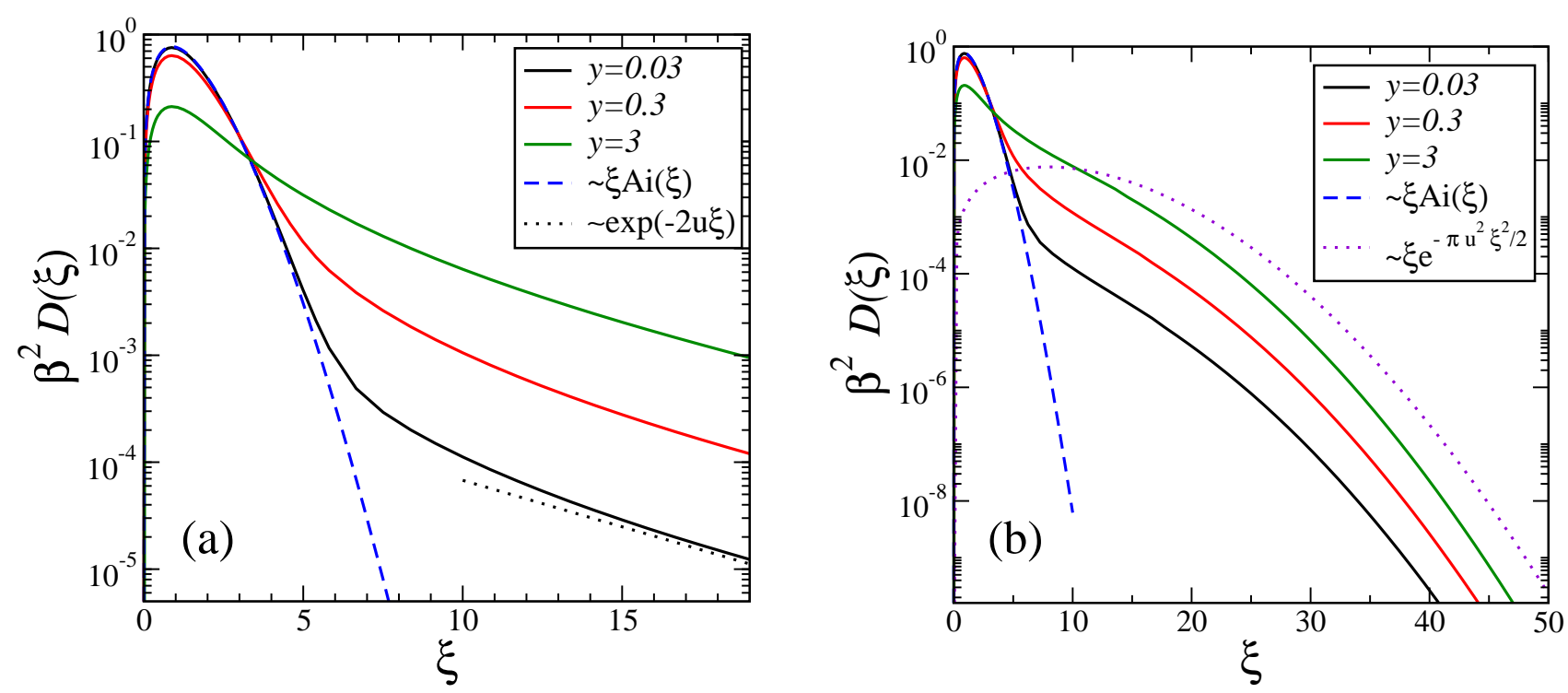

Figure 6: Stationary IPDF of the CDPR with input, for the case $u=0.1$ and several values of $y$. Left panel (a): reset distribution $F(x)=\exp (-c x)$. The IPDFs for diffusion-coagulation with input, both for input without a reset and for independent particles, are also shown. Right panel (b): reset distribution $F(x)=\operatorname{erfc}\left(\frac{1}{2} \sqrt{\pi} c x\right)$. The IPDFs for diffusion-coagulation with input and for diffusion-coagulation are also shown.

\subsection{IPDF with input}

From the previous equation (3.6), the stationary (IPDF) is cast in the scaling form

$$
\mathcal{D}(x):=\frac{1}{2 \rho_{\text {stat }}} \frac{\partial^{2} f(x)}{\partial x^{2}}=\frac{\beta^{2}}{\rho_{\text {stat }}} D(\beta x, c / \beta, \beta \mu)
$$

with the three-variable scaling function

$$
\begin{aligned}
D(\xi, u, y)= & \frac{(\xi+y) \operatorname{Ai}(\xi+y)}{\operatorname{Ai}(y)}-\pi y(\xi+y) \operatorname{Ai}(\xi+y) \frac{\operatorname{Bi}(y)}{\operatorname{Ai}(y)} \int_{0}^{\infty} \mathrm{d} Y F(u Y / c) \operatorname{Ai}(Y+y) \\
& +\pi y(\xi+y) \operatorname{Bi}(\xi+y) \int_{\xi}^{\infty} \mathrm{d} Y F(u Y / c) \operatorname{Ai}(Y+y) \\
& +\pi y(\xi+y) \operatorname{Ai}(\xi+y) \int_{0}^{\xi} \mathrm{d} Y F(u Y / c) \operatorname{Bi}(Y+y) \\
& +\pi y F(u \xi / c)\left[\operatorname{Bi}(\xi+y) \operatorname{Ai}^{\prime}(\xi+y)-\operatorname{Bi}^{\prime}(\xi+y) \operatorname{Ai}(\xi+y)\right]
\end{aligned}
$$

and the scaling variables $\xi:=\beta x, u:=c / \beta$ and $y:=\beta \mu$.

In figure 6, the consequences of the reset are illustrated. First, for a reset to uncorrelated particles, the behaviour seen in the left panel (figure 6a) is qualitatively the same as seen above in the case without input. At short interval sizes, the system has enough time between two resets to build up its natural correlations, so that the shape of the IPDF is essentially given by the Airy function (see table 2) and its stretched-exponential form. For larger sizes, the particles become uncorrelated and the IPDF goes over to a simple exponential.

A similar pattern is seen when resetting to configurations of correlated particles. In the right panel (figure 6b), the IPDF for a reset to a configuration of simple diffusion-coagulation 
with mean density $c$ is shown. With respect to the previous situation, the IPDF of the reset distribution $F(\xi)$ falls off more rapidly for $\xi \gg 1$ than the "natural' one of the underlying process. Yet, we see that the reset rate $r$ again sets a time scale such that for sufficiently small interval sizes, the distribution of the empty intervals is the natural one of diffusion-coagulation with input and goes over to the one put in by the reset for larger intervals 4

In any case, these examples illustrate the subtle nature of the stationary states in simple particle-reaction models with a stochastic reset. The main new feature is a new scale set by the reset rate $r>0$, such that at sufficiently small length scales, the 'natural' correlations of the dynamics dominate whereas at larger length scales, those of the reset configurations become dominant.

\section{Conclusions}

Analysis of the effects of a stochastic reset provides an alternative route to better appreciate the consequences of the breaking of detailed balance. This breaking is required to obtain nonequilibrium stationary states. In the present work, we have studied how the properties of a simple reaction-diffusion model are modified through the introduction of a stochastic reset. This was achieved by identifying a new member in the class of models which may be solved exactly through the 'empty-interval method'.

A particular bonus of this technique is that it provides a very direct access to the distribution of the distances between particles. In this way, we have seen that (i) the model's behaviour is not much affected by the reset at short length scales but (ii) profoundly altered at larger scales. The coexistence of at least two kinds of correlations at different length scales should be identified as the main mechanism which drives the system to a new non-equilibrium stationary state.

Comparison of our analytical results with Monte Carlo simulations permit to identify how to set up analogous studies in different many-body problems and/or networks, where exact analytical results may not be so readily available.

\section{Appendix. Remark on the Monte Carlo simulations}

In order to further illustrate the proper choice of a microscopic model of the CDPR, which for continuous time would be described by (1.2), we compare two different choices for the transition rates in a Monte Carlo simulation:

- Method 1 : as already defined in section 1 in the main text. We insist that the probability $\mathcal{P}_{r}$ chosen for the reset guarantees a reset with probability $r$ per sweep.

- Method 2 : for each sweep of $\mathcal{N}$ individual Monte Carlo steps, the move to be carried out is globally chosen for all particles: either $\mathcal{N}$ usual coagulation-diffusion steps are selected with probability $2 D /(2 D+r)$, or else a global reset is chosen, with probability $r /(2 D+r)$.

\footnotetext{
${ }^{4}$ We did not detect any evidence that for extremely large values of $\xi$, the IPDF would cross over to a simple exponential form.
} 

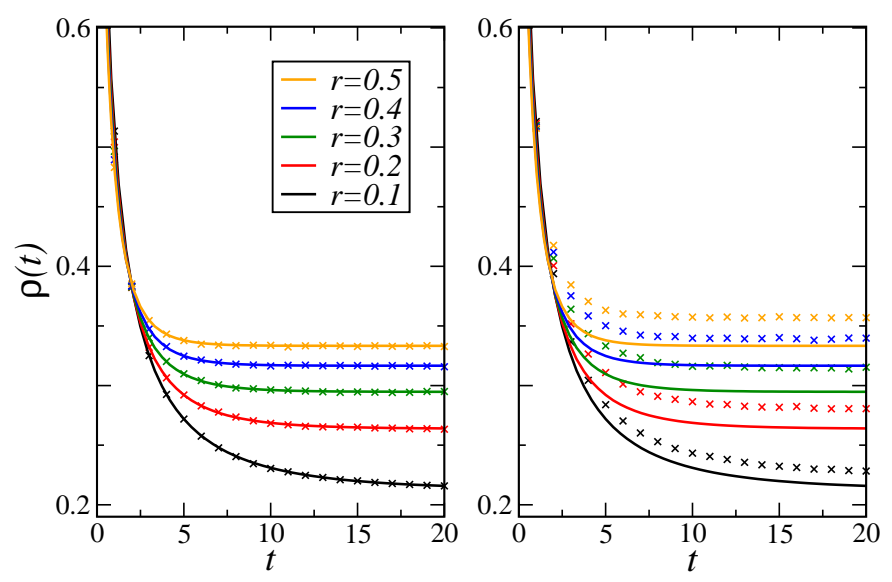

Figure 7: Time-dependent particle-density $\rho(t)$ in two distinct Monte Carlo simulations on a periodic chain with $\mathcal{N}=512$ sites. Left panel: result of method 1, right panel: results of method 2. The parameters used are $D=1 / 2, p=0.5$ and $r=[0.1,0.2,0.3,0.4,0.5]$ from bottom to top. Initially the system was entirely filled. The full lines give the exact analytical result, see section 2 .

In figure 7, the results for the time-dependent density $\rho(t)$ of these choices of the dynamics are shown, for a periodic chain with $\mathcal{N}=512$ sites, $D=\frac{1}{2}$, a reset to uncorrelated particles with mean density $p=0.5$ and several values of $r$. Comparison with the exact result, derived in section 2, shows that while the data obtained from method 1 perfectly agree, there is no agreement with those from method 2 .

Acknowledgements: This work was started during the $5^{\text {th }}$ KIAS conference on Statistical Physics. We thank S.N. Majumdar and J.D. Noh for useful discussions. MH is grateful to KIAS Seoul for warm hospitality. This work was partly supported by the Collège Doctoral franco-allemand Nancy-Leipzig-Coventry (Systèmes complexes à l'équilibre et hors équilibre) of UFA-DFH and also by the Basic Science Research Program through the NRF Grant No. 2013R1A1A2A10009722.

\section{References}

[1] M. Abramowitz and I.A. Stegun, Handbook of Mathematical Functions, Dover (New York 1965)

[2] E. Abad, S. B. Yuste and K. Lindenberg, Phys. Rev. E86, 061120 (2012). [arXiv:1210.1235]

[3] A. Aghamohammadi and M. Khorrami, Eur. Phys. J. B47, 583 (2005). [cond-mat/0511649]

[4] C. Arita, J. Bouttier, P.L. Kaprivsky and K. Mallick, [arXiv:1307.4367].

[5] D. ben Avraham and S. Havlin, Diffusion and Reactions in Fractals and Disordered Systems, Cambridge University Press (Cambridge 2000)

[6] D. ben Avraham, M. Burschka and C.R. Doering, J. Stat. Phys. 60695 (1990).

[7] D. ben Avraham, Phys. Rev. Lett. 81, 4756 (1998).

[8] O. Bénichou, N. Meunier, S. Redner and R. Voituriez, Phys. Rev. E85, 021137 (2012). [arXiv:1110.0348] 
[9] M.A. Burschka, C.R. Doering and D. ben Avraham, Phys. Rev. Lett. 63, 700 (1989).

[10] S.R. Dahmen, J. Phys. A: Math. Gen. 28, 905 (1995). [cond-mat/9405031]

[11] C.R. Doering and M.A. Burschka, Phys. Rev. Lett. 64245 (1990).

[12] C.R. Doering, Physica A188, 386 (1992).

[13] X. Durang, J.-Y. Fortin, D. del Biondo, M. Henkel and J. Richert, J. Stat. Mech. P04002 (2010). [arXiv:1012.4724]

[14] X. Durang, J.-Y. Fortin and M. Henkel, J. Stat. Mech. P02030 (2011). [arXiv:1001.3526]

[15] M.R. Evans and S.N. Majumdar, Phys. Rev. Lett. 106, 160601 (2011). [arXiv:1102.2704]

[16] M.R. Evans and S.N. Majumdar, J. Phys. A Math. Theor. 44, 435001 (2011). [arXiv:1107.4225]

[17] M.R. Evans, S.N. Majumdar and K. Mallick, [arXiv:1212.4096].

[18] J. Franke and S.N. Majumdar, J. Stat. Mech. P05024 (2012). [arXiv:1203.2859]

[19] M. Henkel and H. Hinrichsen, J. Phys. A: Math. Gen. 34, 1561 (2001). [cond-mat/0010062]

[20] M. Henkel, H. Hinrichsen and S. Lübeck, "Non-equilibrium phase transitions vol. 1: absorbing phase transitions", Springer (Heidelberg 2009).

[21] M. Khorrami, A. Aghamohammadi and M. Alimohammadi, J. Phys. A: Math. Gen. 36, 345 (2003). [cond-mat/0112490]

[22] R. Kopelman, C.S. Li and Z.-Y. Shi, J. Lumin. 45, 40 (1990);

J. Prasad and R. Kopelman, Chem. Phys. Lett. 157, 535 (1989).

[23] K. Krebs, M.P. Pfannmüller, B. Wehefritz and H. Hinrichsen, J. Stat. Phys. 78, 1429 (1995). [cond-mat/9402017], [cond-mat/9402018], [cond-mat/9402019]

[24] R. Kroon, H. Fleurent and R. Sprik, Phys. Rev. E47, 2462 (1993).

[25] T. Masser and D. ben-Avraham, Phys. Lett. A275, 382 (2000). [cond-mat/0008448]

[26] T.G. Mattos, C. Meíja-Monasteiro, R. Metzler and G. Oshanin, Phys. Rev. E86, 031143 (2012). [arXiv:1206.1003]

[27] C. Meíja-Monasteiro, G. Oshanin and G. Schehr, J. Stat. Mech. P06022 (2011). [arXiv:1106.4182]

[28] M. Montero and J. Villaroel, [arXiv:1206.4570].

[29] R. Munasinghe, R. Rajesh, R. Tribe and O. Zaboronski, Comm. Math. Phys. 268, 717 (2006).

[30] R. Munasinghe, R. Rajesh and O. Zaboronski, Phys. Rev. E73, 051103 (2006). [cond-mat/0506398]

[31] R.M. Russo, E.J. Mele, C.L. Kane, I.V. Rubtsov, M.J. Therien and D.E. Luzzi, Phys. Rev. B74 041405(R) (2006).

[32] P.-A. Rey and M. Droz, J. Phys. A: Math. Gen. 30, 1101 (1997). [cond-mat/9609088]

[33] L.P. Sanders and T. Ambjörnsson, [arXiv:1205.1931]. 
[34] A. Srivastava and J. Kuno, Phys. Rev. B79, 205407 (2009).

[35] J.L. Spouge, Phys. Rev. Lett. 60, 871 (1988); erratum Phys. Rev. Lett. 601885 (1988).

[36] Z. Rácz, Phys. Rev. Lett. 55, 1707 (1985).

[37] R. Zia and B. Schmittmann, J. Stat. Mech. P07012 (2007). [cond-mat/0701763]. 